Int. J. Dev. Biol. 50: 561-569 (2006)

doi: $10.1387 / \mathrm{ijdb} .062151 \mathrm{cc}$

Technical Article

\title{
Transient expression of apoaequorin in zebrafish embryos: extending the ability to image calcium transients during later stages of development
}

\author{
CHRIS Y. CHEUNG ${ }^{1}$, SARAH E. WEBB ${ }^{1}$, ANMING MENG ${ }^{2}$ and ANDREW L. MILLER ${ }^{*}, 1$ \\ ${ }^{1}$ Department of Biology, The Hong Kong University of Science and Technology, Hong Kong SAR, People's Republic of China and \\ ${ }^{2}$ Department of Biological Sciences and Biotechnology, Tsinghua University, Beijing 100084, People's Republic of China.
}

\begin{abstract}
When aequorin is microinjected into cleavage-stage zebrafish embryos, it is largely used up by $\sim 24$ hours. Thus, it is currently not possible to image $\mathrm{Ca}^{2+}$ signals from later stages of zebrafish development using this approach. We have, therefore, developed protocols to express apoaequorin, i.e., the protein component of aequorin, transiently in zebrafish embryos and then reconstitute intact aequorin in vivo by loading the coelenterazine co-factor into the embryos separately. Two types of apoaequorin mRNA, aeq-mRNA and aeq::EGFP-mRNA, the latter containing the enhanced green fluorescent protein (EGFP) sequence, were in vitro transcribed and when these were microinjected into embryos, they successfully translated apoaequorin and a fusion protein of apoaequorin and EGFP (apoaequorin-EGFP), respectively. We show that aeq::EGFP mRNA was more toxic to embryos than equivalent amounts of aeq-mRNA. In addition, in an in vitro reconstitution assay, apoaequorin-EGFP produced less luminescence than apoaequorin, after reconstitution with coelenterazine and with the addition of $\mathrm{Ca}^{2+}$. Furthermore, when imaging intact coelenterazine-loaded embryos that expressed apoaequorin, $\mathrm{Ca}^{2+}$ signals from $\sim 2.5$ to $48 \mathrm{hpf}$ were observed, with the spatio-temporal pattern of these signals up to $24 \mathrm{hpf}$, being comparable to that observed with aequorin. This transient aequorin expression approach using aeq-mRNA provides a valuable tool for monitoring $\mathrm{Ca}^{2+}$ signaling during the 24-48 hpf period of zebrafish development. Thus, it effectively extends the aequorin-based $\mathrm{Ca}^{2+}$ imaging window by an additional 24 hours.
\end{abstract}

KEY WORDS: aequorin, apoaequorin, coelenterazine, $\mathrm{Ca}^{2+}$, zebrafish

\section{Introduction}

Calcium ions $\left(\mathrm{Ca}^{2+}\right)$ are ubiquitous second messengers that are known to control a wide variety of biological processes, such as gene transcription, cytoskeletal remodeling, membrane trafficking, cytokinesis and cell movement (reviewed by Berridge et al., 2003). Via combinations of these processes it has also long been suggested that $\mathrm{Ca}^{2+}$ signaling, in the form of pulses, waves and steady gradients, might play a key role in pattern formation during embryogenesis, from the earliest stages of development to the differentiation of specific cell types (reviewed by Webb and Miller, 2003). Whereas the intracellular $\mathrm{Ca}^{2+}$ concentration is relatively low in resting cells (i.e., $\sim 60-100 \mathrm{nM}$ ), it can increase to $\sim 1 \mu \mathrm{M}$ or more, in a short period of time when cells are stimulated to perform particular functions (Bootman et al., 2001). Thus, by changing the dimensions of cytosolic $\mathrm{Ca}^{2+}$ events in terms of space, time and amplitude, information can be rapidly and efficiently exchanged both across and between cells (Berridge et al., 2003).

One of the first reporters used to monitor intracellular $\mathrm{Ca}^{2+}$ levels in living cells was the bioluminescent $\mathrm{Ca}^{2+}$-sensitive protein complex, aequorin (Ridgway and Ashley, 1967). Originally isolated from the luminescent jellyfish Aequorea aequorea (Shimomura et al., 1962), the aequorin complex is made up from an apo-protein (apoaequorin) of $\sim 21 \mathrm{kDa}$ that contains four helix-loop-helix "EFhand" domains (three of which can bind to $\mathrm{Ca}^{2+}$; Head et al., 2000), a prosthetic co-factor of $\sim 420 \mathrm{Da}$ (coelenterazine) and $\mathrm{O}_{2}$, in the form of peroxide (Shimomura and Johnson, 1978). In the presence of free $\mathrm{Ca}^{2+}$, the coelenterazine is oxidized to coelenteramide, with an associated release of $\mathrm{CO}_{2}$ and blue light (at $\sim 470 \mathrm{~nm}$ ) is produced (Shimomura and Johnson, 1973). Following this reaction, the aequorin complex is effectively "spent" and active aequorin

Abbreviations used in this paper: $\mathrm{Ca}^{2+}$, calcium ions; EGFP, enhanced green fluorescent protein; hpf, hours post-fertilization; RLU, relative light units

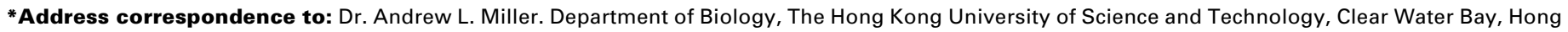
Kong SAR, People's Republic of China. Fax: +852-2358-1559. e-mail: almiller@ust.hk
} 
A

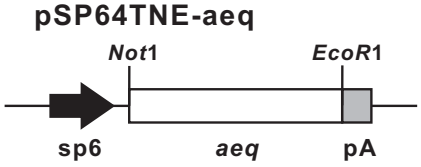

B

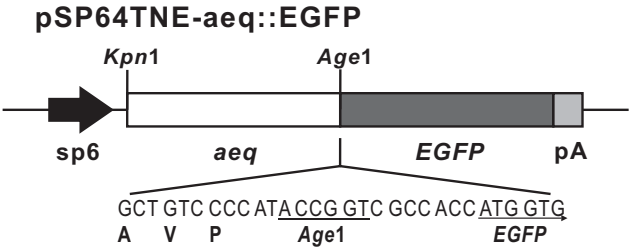

Fig. 1. Map of the pSP64TNE-aeq and pSP64TNE-aeq::EGFP plasmids. (A) For pSP64TNE-aeq, the full length sequence of aeq (591 bp) was cloned in the pSP64TNE plasmid, while (B) for pSP64TNE-aeq::EGFP, the aeq sequence, minus its stop codon was ligated in-frame to the $5^{\prime}$ end of EGFP to make a fusion protein of apoaequorin and EGFP in pSP64TNE. Black line, pSP64TNE plasmid vector sequences; sp6 (black arrow), sp6 promoter sequence; aeq, apoaequorin cDNA sequence; EGFP, enhanced green fluorescent protein sequence; $P A$, polyadenylation signal.

cannot be regenerated unless additional coelenterazine is provided (Shimomura and Johnson, 1975). In living cells, aequorin has a wide dynamic range (i.e., $0.1-100 \mu \mathrm{M} \mathrm{Ca}^{2+}$ ), where the light output of the aequorin luminescence reaction is approximately proportional to the square of the free $\mathrm{Ca}^{2+}$ concentration and thus it shows inherent contrast enhancement (Shimomura and Inouye, 1996). In addition, $\mathrm{Ca}^{2+}$-bound aequorin emits light without excitation, thus the autofluorescence and photo-induced cytotoxicity that can take place with fluorescent $\mathrm{Ca}^{2+}$ reporters, does not occur with aequorin. Aequorin has also been shown not to interfere with the normal development of living embryos (Miller et al., 1994). This allows for long-term $\mathrm{Ca}^{2+}$ imaging, which is particularly suited for studying

A

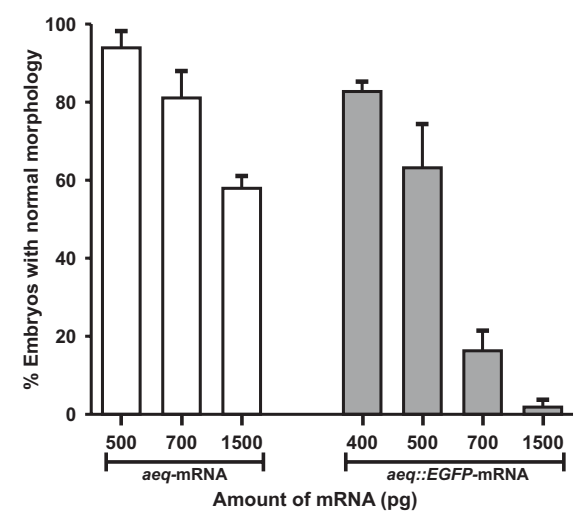

Fig. 2. Effect of aeq-mRNA and aeq::EGFP-mRNA on normal development. Embryos were injected with different amounts of aeq-mRNA or aeq::EGFP-mRNA at the 1-cell stage and then observed after 24 h. (A) Bar graph to illustrate the percentage of normal embryos that developed for each amount of $m R N A$ injected. The data are expressed as mean \pm SEM $(n \geq 3)$. (B) Bright-field images to show the effect of injecting different amounts of aeq-mRNA and aeq::EGFP-mRNA into embryos compared to (i) an uninjected control embryo at $24 \mathrm{hpf}$. Representative examples of embryos injected with (ii) 500 pg, (iii) 700 pg or (Iv) 1500 pg aeq-mRNA, or with (v) 400 pg, (vi) 700 pg or (vii) 1500 pg aeq::EGFP-mRNA. Embryos injected with $>700$ pg aeq-mRNA or $>400$ pg aeq::EGFP-mRNA exhibited defects in the development of both head and tail. Scale bar, $200 \mu \mathrm{m}$. embryonic development (Miller et al., 1994; Ashworth and Brennan, 2005).

One of the current popular vertebrate models for studying embryogenesis in recent years is the zebrafish (Danio rerio). A combination of the optical transparency, large size and rapid development of their embryos makes them particularly ideal for $\mathrm{Ca}^{2+}$ imaging. Through the microinjection of aequorin into the blastoderm of zebrafish embryos at the single-cell stage and the use of a custom-designed Photon Imaging Microscope System (PIMS), we have been able to continuously image the $\mathrm{Ca}^{2+}$ signals from zebrafish embryos, beginning at egg activation (Lee et al., 1999), then through ooplasmic segregation and cytokinesis in the early Cleavage Period (Webb et al., 1997; Leung etal., 1998), during the Gastrula (Gilland etal., 1999) and Segmentation (Webb and Miller, 2003) Periods and up to around the prim- 6 stage around the start of the Pharyngula Period. Up until now, however, we have not been able to image $\mathrm{Ca}^{2+}$ signals in embryos at later stages of development. This is because aequorin that is injected into embryos at the one-cell stage and becomes partitioned into daughter cells by subsequent cell divisions, is used up by $\sim 24$ hours. It is also clearly impractical to microinject the reporter into each of the tens of thousands of cells that comprise these later-stage embryos. One way to get around this restriction is to express the protein component of aequorin (i.e., apoaequorin), transgenically in zebrafish and then to reconstitute the active protein complex in vivo by loading the coelenterazine co-factor into the embryos separately. The techniques used for transforming apoaequorin cDNA into single cell organisms (e.g. Escherichia colf; Inouye et al., 1989) and some simple multicellular organisms (e.g. the slime mold, Dictyostelium discoideum, Saran etal., 1994), as well as in various mammalian tissue culture cells (Button and Brownstein, 1993), have already been successfully developed. Active aequorin has then been reconstituted within these transgenic cells, tissues or whole organisms via a very straightforward incubation in coelenterazine solution. To date, however, there is just a single

B
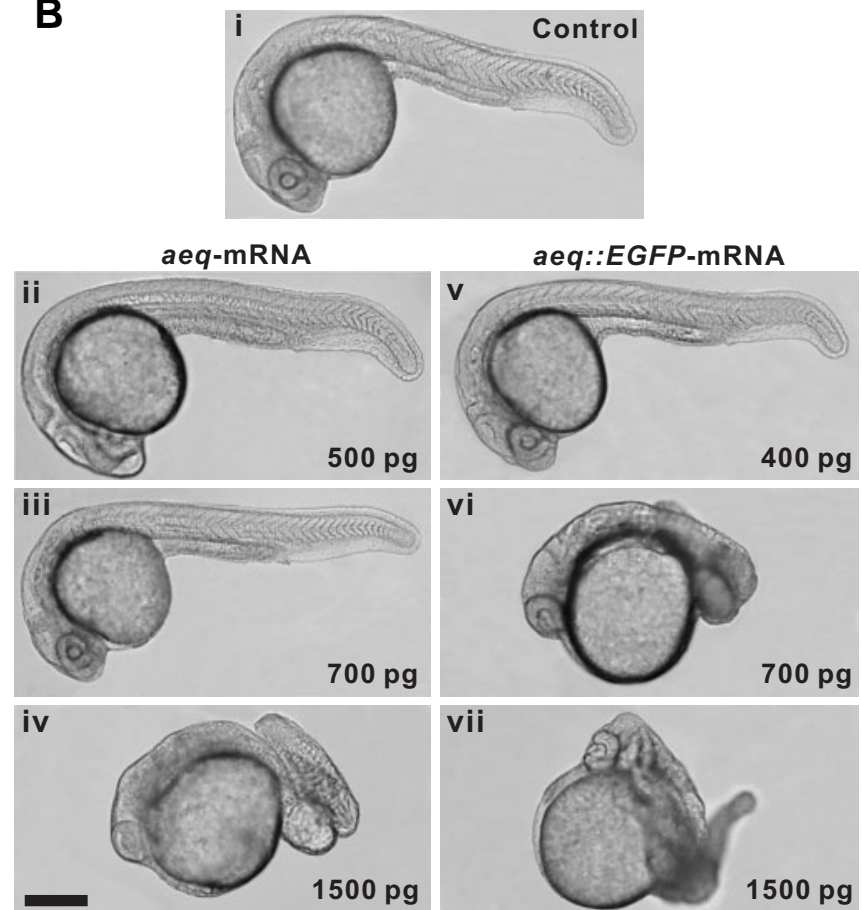
recent report that describes the injection of cDNA and also mRNA apoaequorin constructs into zebrafish embryos (Ashworth and Brennan, 2005). The authors also highlight the challenges in detecting $\mathrm{Ca}^{2+}$ signals in the apoaequorin expressing fish and discuss some of the problems in loading coelenterazine into later-stage embryos for the generation of active aequorin.

In this study, therefore, we expand on the work reported by Ashworth and Brennan (2005) and describe in detail, protocols developed to express apoaequorin transiently and ubiquitously in zebrafish embryos, via the injection of apoaequorin mRNA followed by incubation with coelenterazine to reconstitute active aequorin in vivo. We demonstrate that $\mathrm{Ca}^{2+}$ signals can be observed from $\sim 2.5$ to $48 \mathrm{hpf}$ and we show that the spatio-temporal pattern of these signals up to $24 \mathrm{hpf}$ is comparable to that observed through injecting single cell-stage embryos with recombinant aequorin. The advantage of this new technique, however, is the additional time (i.e., from 24 to $48 \mathrm{hpf}$ ) that it extends the noninvasive imaging window. We suggest, therefore, that it will be a useful interim methodology for $\mathrm{Ca}^{2+}$ imaging until such time as stable transgenic lines of apoaequorin-expressing fish are developed. The extension of the aequorin-based imaging period through the Pharyngula Period (24 to $48 \mathrm{hpf}$ ) to the beginning of the Hatching Period (48 to 72 hpf) is significant, as it will thus encompass the important developmental events of the former. These include the appearance of tactile sensitivity; the coordination of myotome contraction resulting in rhythmic bouts of swimming; the development of the circulatory system and the initiation of the heart-beat (Kimmel et al., 1995). These events may require different forms of $\mathrm{Ca}^{2+}$ signaling, both for their development and their function. We propose, therefore, that a transient apoaequorin expressing zebrafish may provide a first step in investigating possible $\mathrm{Ca}^{2+}$ related signaling pathways and their down-stream targets.

\section{Results and Discussion}

In this study, two types of apoaequorin mRNA, i.e., aeq-mRNA and aeq.:EGFP-mRNA, were in vitro transcribed using the corresponding pSP64TNE-aeq and pSP64TNE-aeq::EGFP plasmids. On their subsequent microinjection into 1-cell stage embryos, they were successfully translated into apoaequorin and an apoaequorinEGFP fusion protein, respectively.

\section{Testing mRNA toxicity}

It has been reported that injection of high concentrations of mRNA can lead to abnormal development and may indeed be lethal to zebrafish embryos (Gilmour et al., 2002). Thus, the maximum amount of aeq-mRNA and aeq ::EGFP-mRNA that could be loaded into zebrafish embryos and still result in normal development was determined. Embryos were injected into the blastodisc at the 1-cell stage with increasing amounts of these mRNAs, after which they were examined for normal development (i.e., by comparison with uninjected control embryos), at $24 \mathrm{hpf}$ (see Fig. 2). Fig. 2A shows that as much as $700 \mathrm{pg}$ aeq-mRNA could be injected into embryos and more than $80 \%$ (i.e., $81.0 \pm 7.0 \%$ ) developed normally, when examined at $24 \mathrm{hpf}$. Indeed, when injected with 500 pg aeq-mRNA, $\sim 94.0 \pm 4.0 \%$ embryos were normal after 24 hours. When the amount of mRNA was increased to $1500 \mathrm{pg}$ per embryo, however, the number of normal embryos observed decreased to $\sim 60 \%$. The aeq::EGFP-mRNA also showed a dose-dependent increase in toxicity in embryos, however, $>80 \%$ normal development (i.e., $83.0 \pm 2.5 \%$ ) could only be achieved when embryos were injected with just $400 \mathrm{pg}$ mRNA. At $500 \mathrm{pg}$ aeq::EGFP. mRNA, the number of normal embryos after 24 hours was just 63 $\pm 11 \%$ and amounts of $700 \mathrm{pg}$ and $1500 \mathrm{pg}$ produced only $16 \pm 5 \%$ and $2 \pm 2 \%$ normal embryos, respectively.

The effect of injecting increasing amounts of aeq-mRNA and aeq::EGFP-mRNA on development is also shown in the bright-field images in Fig. 2B. When embryos were injected with $700 \mathrm{pg}$ aeq mRNA or $400 \mathrm{pg}$ aeq::EGFP-mRNA, their gross morphology after 24 hours was comparable to the uninjected controls. However, when embryos were injected with more than either $700 \mathrm{pg}$ aeqmRNA or $400 \mathrm{pg}$ aeq::EGFP-mRNA, a range of abnormalities was observed. These included defects in the development of the head and tail. The reasons for $m R N A$-induced abnormal development or death are thought to be both varied and complex, but are suggested to be at least in part, due to the extra demand on the proteinmaking resources of the embryo (Gilmour et al., 2002). In subsequent experiments, $700 \mathrm{pg}$ aeq-mRNA and $400 \mathrm{pg}$ aeq::EGFPmRNA were used.

\section{Expression of EGFP in the aeq::EGFP-mRNA injected embryo}

The aeq::EGFP-mRNA was specifically prepared in order to test whether the expression of EGFP could be used to provide spatio-
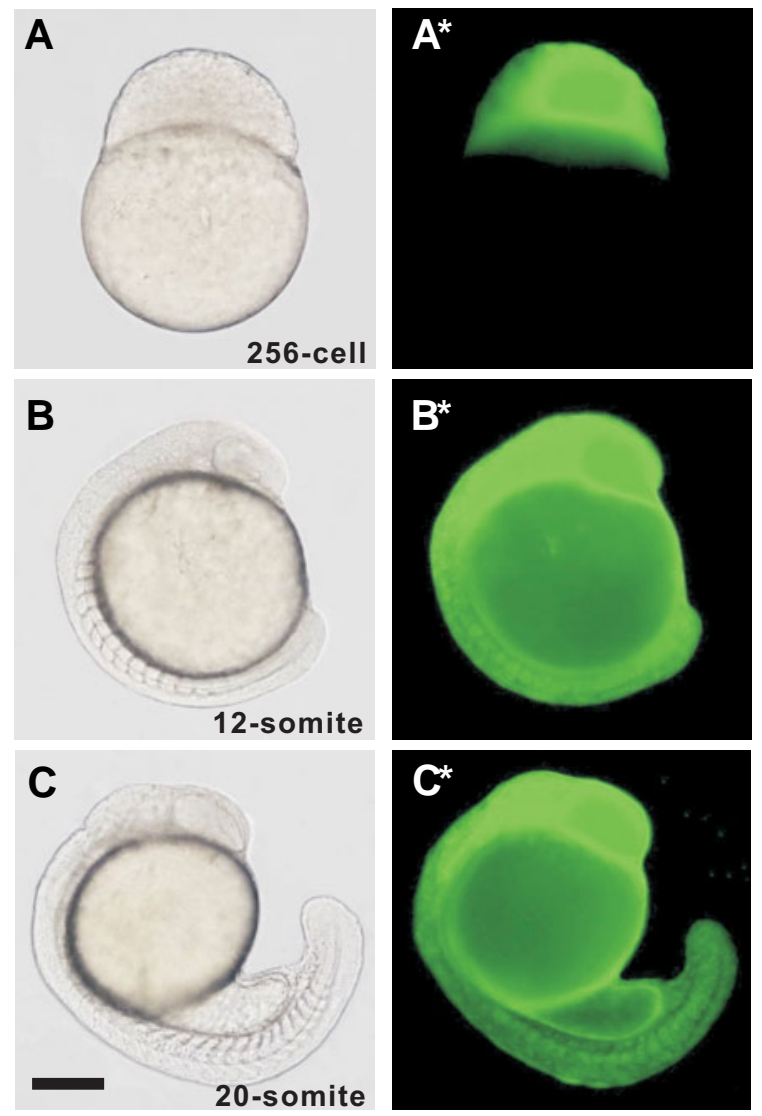

Fig. 3. Expression of enhanced green fluorescent protein (EGFP) in the aeq::EGFP-mRNA injected embryos. $(\mathbf{A}, \mathbf{B}, \mathbf{C})$ Bright-field and $\left(\mathbf{A}^{*}, \mathbf{B}^{*}, \mathbf{C}^{*}\right)$ respective EGFP fluorescence images of a representative aeq::EGFP-mRNA injected embryo at the $\left(A, A^{*}\right)$ 256-cell, $\left(B, B^{*}\right)$ 12somite and $\left(C, C^{*}\right)$ 20-somite stages. Scale bar,200 $\mu \mathrm{m}$. 
temporal information about the transiently expressed apoaequorin in the developing embryo. GFP has been successfully tagged to other proteins in a variety of organisms, including zebrafish (reviewed by March et al., 2003). Thus, embryos were injected with $400 \mathrm{pg}$ aeq::EGFP-mRNA into the blastodisc at the 1-cell stage and fluorescence images were acquired at different stages of development. Figure 3 illustrates the pattern and level of expression of EGFP in a representative aeq::EGFP-mRNA injected embryo at the 256-cell ( 2.5 hpf), 12-somite ( $15 \mathrm{hpf})$ and 20 -somite stages ( $19 \mathrm{hpf})$. An intense and ubiquitous pattern of EGFP fluorescence is observed at all the stages shown. This result is not unexpected, having been reported previously for a similar GFP-apoaequorin mRNA-based probe (Ashworth and Brennan, 2005).

\section{Time course of apoaequorin expression and estimation of expression levels}

Embryos were injected with either $700 \mathrm{pg}$ aeq-mRNA or $400 \mathrm{pg}$ aeq::EGFP-mRNA into the blastodisc at the 1-cell stage and Western blot analysis was conducted to estimate the level of apoaequorin expression at different time points up to $30 \mathrm{hpf}$ (Fig. 4). Representative examples $(n=3)$ of the time course of apoaequorin expression following aeq-mRNA or aeq.:EGFPmRNA injection are shown in Fig. 4B and 4C, respectively. These expression levels were quantified and then normalized using recombinant aequorin as an internal positive control, before graph plotting.

The graph (Fig. 4A) demonstrates that when embryos were injected with either aeq-mRNA or aeq::EGFP-mRNA, the expression of apoaequorin could be detected as early as $2 \mathrm{hpf}$ and reached a peak level at $4 \mathrm{hpf}$, after which it started to decline. This drop in the level of apoaequorin expression was more rapid in the aeq-mRNA-injected embryos. At $30 \mathrm{hpf}$, for example, no apoaequorin was detected in the aeq-mRNA injected embryos, whereas it was still detected at nearly half the maximum level in the aeq ::EGFP-mRNA injected embryos at this time. The lower level of apoaequorin expression that is shown in the graph (Fig. 4A) for the aeq:EGFP-mRNA injected embryos, might simply be due to the lower amount of mRNA injected (i.e., $400 \mathrm{pg}$ per embryo, as opposed to $700 \mathrm{pg}$ ).

In order to estimate the concentrations of apoaequorin and apoaequorin-EGFP protein expressed, the bands from three separate Western blot experiments for each expression vector (including those shown in Fig. 4 B,C) were quantified by comparing them to an internal positive control lane loaded with $20 \mathrm{pg}$ of aequorin. The mean values obtained from three separate experiments indicated that approximately $83.3 \mathrm{pg}$ of apoaequorin and $34.2 \mathrm{pg}$ of apoaequorin-EGFP were expressed in embryos injected with aeq-mRNA or aeq::EGFP-mRNA, respectively. Assuming the volume of a zebrafish embryo is $\sim 128 \mathrm{nl}$ and approximately $68 \%$ of this is water (Leung et al., 1998), at $4 \mathrm{hpf}$ this equates to around $45.6 \mathrm{nM}$ of apoaequorin and $18.7 \mathrm{nM}$ of apoaequorin-EGFP, respectively.

Thus, the results of these experiments indicate that even though aeq::EGFP-mRNA was more toxic to embryos than equivalent amounts of aeq-mRNA, both mRNAs were successful in translating apoaequorin and apoaequorin-EGFP, respectively in embryos. We thus proceeded to determine whether these proteins had luminescent capability on reconstitution with $f$ coelenterazine followed by the addition of $\mathrm{Ca}^{2+}$.

\section{A}

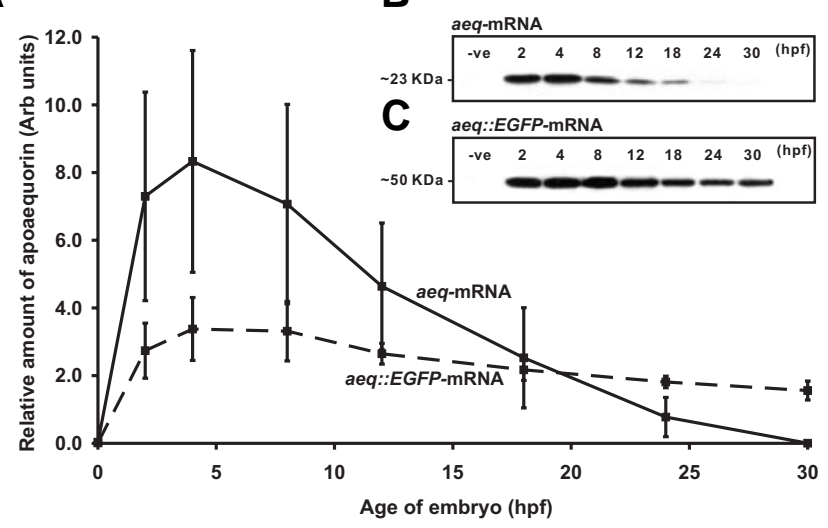

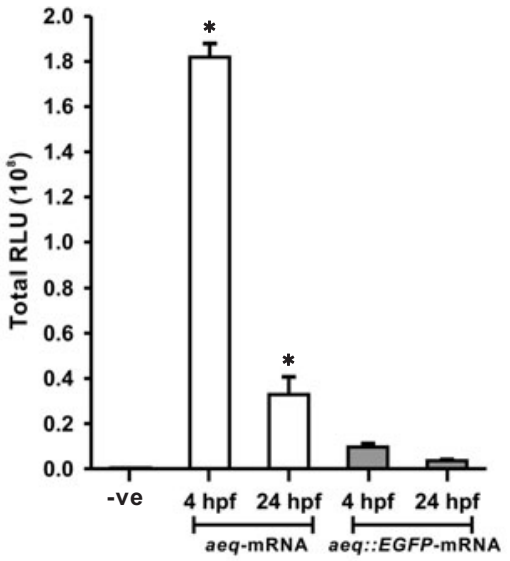

Fig. 4 (Left column). Apoaequorin expression profiles of aeq-mRNA and aeq::EGFP-mRNA injected embryos. A time-course Western blot analysis was conducted using embryos that had been injected with either aeq-mRNA or aeq::EGFP-mRNA at the single cell stage and then embryo extract prepared at 2, 4, 8, 12, 18, 24 and $30 \mathrm{hpf}$. (A) The line graph illustrates the mean $\pm S E M(n=3)$ level of apoaequorin expression in extract from embryos injected with $700 \mathrm{pg}$ aeq-mRNA or 400 pg aeq::EGFP-mRNA, relative to the internal control, aequorin. (B,C) Representative examples of Western blots to show the relative level and temporal expression pattern of apoaequorin in extract that was prepared from embryos injected with (B) aeq-mRNA or (C) aeq::EGFP-mRNA. Extract prepared from 2 embryos was loaded into each lane.

Fig. 5 (Right column). Comparison of the total luminescence from embryo extract, prepared from embryos that had been injected with 700 pg aeq-mRNA or $\mathbf{4 0 0} \mathbf{~ p g ~ a e q : : E G F P - m R N A ~ a t ~ t h e ~ 1 - c e l l ~ s t a g e . ~ A e q u o r i n ~ w a s ~ r e c o n s t i t u t e d ~ f r o m ~ a p o a e q u o r i n ~ i n ~ t h e ~ e x t r a c t ~ b y ~ i n c u b a t i o n ~ w i t h ~} \mathrm{f}$ coelenterazine, after which $\mathrm{CaCl}_{2}$ was added at $4 \mathrm{hpf}$ and $24 \mathrm{hpf}$ and the resulting luminescence quantified. The data are expressed as mean \pm SEM total relative light units (RLU) of 3 independent experiments. The RLU from each experiment represents the total luminescence produced from an extract obtained from $10 \mathrm{mRNA}$-injected embryos in $1 \mathrm{~min}$ immediately after the addition of $\mathrm{Ca}^{2+} .{ }^{*}$ Indicates measurements that are statistically significant at $P<0.01$ using one-way ANOVA (parametric) and Newman-Keuls multiple comparison test. 


\section{Comparative in vitro aequorin reconstitution assay of extract prepared from aeq-mRNA injected embryos}

The amount of luminescence produced by embryo extract, which had been prepared from embryos that had been injected with $700 \mathrm{pg}$ aeq-mRNA or $400 \mathrm{pg}$ aeq::EGFP-mRNA was compared (Fig. 5). The amount of luminescence produced by embryos that were not injected with mRNA but that had been treated with $f$-coelenterazine at $4 \mathrm{hpf}$ is shown in lane 1 (labeled '-ve') as a negative control. Figure 5 shows that the level of luminescence produced at both $4 \mathrm{hpf}$ and $24 \mathrm{hpf}$ in aeq-mRNA injected embryos was significantly greater than that produced at the same time points in the aeq::EGFP-mRNA injected embryos, respectively. For example, at $4 \mathrm{hpf}$, the luminescence produced from the aeqmRNA injected embryos was $\sim 18$-fold higher than that produced by the aeq::EGFP-mRNA injected embryos at the same time (i.e., $1.82 \pm 0.06 \times 10^{8} \mathrm{RLU}$ compared to $\left.0.1 \pm 0.02 \times 10^{8} \mathrm{RLU}\right)$. In addition, the luminescence produced from aeq-mRNA injected embryos at $4 \mathrm{hpf}$ was $\sim 5.5$-fold higher than that from aeq-mRNA injected embryos at $24 \mathrm{hpf}$ (i.e., $1.82 \pm 0.06 \times 10^{8} \mathrm{RLU}$ compared to $\left.0.33 \pm 0.08 \times 10^{8} \mathrm{RLU}\right)$. RLU values represent the amount of light collected for $1 \mathrm{~min}$ immediately after the addition of $200 \mu \mathrm{l}$ of $150 \mathrm{mM} \mathrm{Ca}{ }^{2+}$. In vitro control experiments indicated that $1 \mathrm{ng}$ of recombinant faequorin produces $\sim 3.92 \times 10^{8} \mathrm{RLU}$ (data not shown as it would over-extend the scale on the y-axis of Fig. 5). Using this number, we can estimate the approximate amounts of $f$-aequorin and $f$-aequorin-EGFP reconstituted at various times from their respective RLU outputs. These comparisons suggest that $\sim 463.1 \mathrm{pg}$ of $f$-aequorin and $\sim 23.7 \mathrm{pg}$ of $f$-aequorin-EGFP were reconstituted at $4 \mathrm{hpf}$ in aeq-mRNA and aeq::EGFP-mRNA injected embryos, respectively. Using the same assumptions for embryo volume and water content indicated earlier (Leung et al., 1998 ) this indicates a final concentration of functional $f$ aequorin of $\sim 254 \mathrm{nM}$ and $f$-aequorin-EGFP of $\sim 13 \mathrm{nM}$ at $4 \mathrm{hpf}$. Our estimate for in vitro faequorin reconstitution is thus similar to the $110 \mathrm{nM}$ suggested previously, to be required to image resting levels of $\mathrm{Ca}^{2+}$ in zebrafish embryos (Créton et al., 1997). However, the luminescent-based value for reconstituted faequorin is $\sim 5$-fold higher than that estimated for apoaequorin protein expression derived from the Western blot protein quantification. On the other hand, the comparative values for apoaequorin-GFP expression and fapoaequorin-EGFP reconstitution are somewhat similar (i.e., comparing $34.25 \mathrm{pg}$ with $23.7 \mathrm{pg}$, respectively). Along with the inherent inaccuracies that accompany these quantification techniques, one additional reason for this large difference with regards to comparing apoaequorin expression with faequorin reconstitution might be attributed to the protein stabilization effect conferred by the covalent bonding of apoaequorin to $f$ coelenterazine. In the case of apoaequorin-EGFP, the fusion of the EGFP portion might in itself confer additional resistance to protein degradation and as a result, there is little difference between the values obtained via these two techniques.

We suggest that the low luminescent ability of the apoaequorinEGFP might be due to the fact that in the preparation of the pSP64TNE-aeq::EGFP plasmid, the EGFP sequence was linked to the $C$-terminus of the apoaequorin sequence. As the apoaequorin C-terminal tail is known to play a key role in binding coelenterazine, the addition of the EGFP sequence in this region might result in a reduced level of coelenterazine binding (Head et al., 2000). We suggest that linking the EGFP sequence to the $\mathrm{N}$-terminus rather
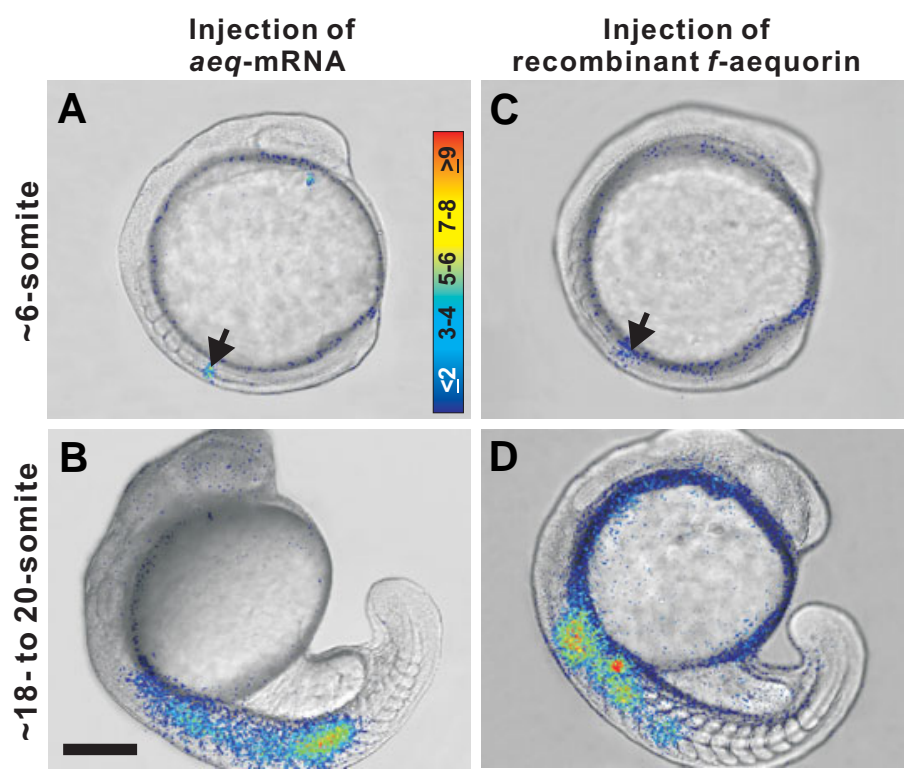

Fig. 6. A comparison of the intracellular $\mathrm{Ca}^{2+}$ signaling patterns observed in representative $(A, B)$ aeq-mRNA injected embryos and $(C, D) f$-aequorin injected embryos during the Segmentation Period. (A,B) Embryos were injected with aeq-mRNA at the single-cell stage and then bathed with $\mathrm{f}$-coelenterazine to reconstitute aequorin from apoaequorin. (C,D) Embryos were injected into the top of the yolk with $\mathrm{f}$-aequorin during the Cleavage Period. $(A, C)$ are trunk signals observed at $\sim 6$-somite stage, during early segmentation, while $(B, D)$ are trunk signals that occur during later segmentation, at $\sim 18$ to 20-somite stage. Each panel represents $60 \mathrm{sec}$ of accumulated light. Color scale indicates luminescent flux in photons/pixel. Scale bar, $200 \mu \mathrm{m}$.

than the C-terminus of apoaequorin might improve the luminescence ability. Indeed, Baubet et al. (2000) have shown that this type of plasmid design (for a fusion protein consisting of GFP and apoaequorin) works well in mammalian cells.

When we introduce recombinant faequorin into single cellstage zebrafish embryos, our normal procedure is to inject approximately $3.5 \mathrm{nl}$ of a $1 \%$ faequorin solution (Webb et al., 1997). This represents a final concentration of faequorin within the embryo of approximately $18 \mu \mathrm{M}$. This is approximately 70 -fold more than the final concentration calculated at the $4 \mathrm{hpf}$ expression and reconstitution peak (i.e., $254 \mathrm{nM}$ ). Créton et al., however, have reported that the estimated in vivo half-life of $h$ aequorin (a semi-synthetic aequorin similar to faequorin; Shimomura et al., 1989) is only around 3 hours (Créton et al., 1997). In addition, it is clear from Fig. 8 that although injection of recombinant $f$ aequorin may initially load an embryo with a substantially greater amount of aequorin, for longer term imaging (i.e., post $24 \mathrm{hpf}$ ) transiently expressed apoaequorin recombined with $f$ coelenterazine does offer a significant advantage.

\section{In vivo reconstitution of aequorin in aeq-mRNA injected intact zebrafish embryos}

Because of the reduced luminescence output of apoaequorinEGFP, the aeq-mRNA injected embryos alone were used for the in vivo reconstitution experiment. Thus, dechorionated embryos that had been injected with $700 \mathrm{pg}$ aeq-mRNA at the 1-cell stage, were incubated in $f$-coelenterazine at the 64 -cell stage (i.e., $2 \mathrm{hpf}$ ) 
$\sim 18$ - to 20-somite
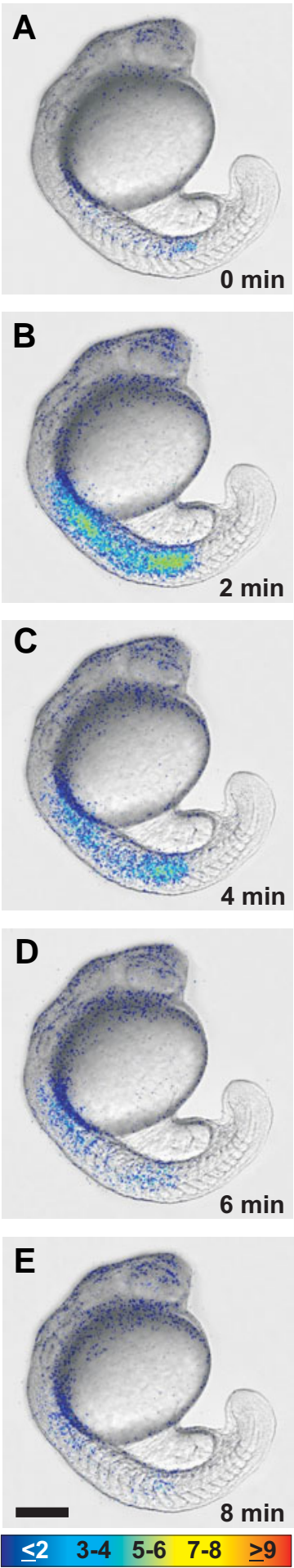

and then the spatial and temporal pattern of $\mathrm{Ca}^{2+}$ signals (as determined by changes in luminescence) produced by these embryos was recorded using our PIMS (Fig. 6). The 64-cell stage was chosen as the starting point for the coelenterazine incubation both because (i) the embryos had started to express apoaequorin (Fig. 4) and (ii) the diffusion of coelenterazine into the embryo was considered to be still relatively efficient as a significant number of blastomeres were still in contact with the bathing medium at this stage. The incubation of embryos at earlier stages was not thought to be of any advantage as apoaequorin was not being expressed and so the potential for coelenterazine to be exposed to oxidizing agents (prior to being protected through binding with apoaequorin; Shimomura, 1995; Head et al., 2000), was considered to be greater. The coelenterazine analogue, $f$-coelenterazine was chosen specifically as it has the same half-life as native coelenterazine, however, once it is reconstituted into the aequorin complex, it produces almost 20 -fold higher luminescence in the presence of $\mathrm{Ca}^{2+}$ (Shimomura, 1991). Furthermore, fcoelenterazine has been reported to have the highest permeability in cells (Shimomura, 1997).

When aeq-mRNA injected embryos were imaged with our PIMS, $\mathrm{Ca}^{2+}$ signals were first detected at $\sim 2.5 \mathrm{hpf}$, after approximately 30-60 minutes incubation in coelenterazine solution (data not shown) and they were observed for up to 48 hours. This clearly demonstrates that transiently expressed apoaequorin can successfully be reconstituted to aequorin in zebrafish embryos in vivo. Furthermore, the $\mathrm{Ca}^{2+}$ signals observed from the 256 -cell stage ( $\sim 2.5 \mathrm{hpf})$ to

Fig. 7. The localized intercellular $\mathrm{Ca}^{2+}$ signals that are generated in the trunk of the representative embryo in Fig. 6B are shown in greater detail. This embryo had been injected with aeq-mRNA at the single-cell stage and then bathed with $\mathrm{f}$-coelenterazine to reconstitute aequorin from apoaequorin. Each panel represents $60 \mathrm{sec}$ of aequorin generated light with a $120 \mathrm{~s}$ gap between each image. Color scale indicates luminescent flux in photons/pixel. Scale bar, $200 \mu \mathrm{m}$. the prim-5 stage ( $48 \mathrm{hpf}$ ) in aeq-mRNA injected embryos were similar to those observed when f-aequorin had been microinjected into early stage embryos. Figure 6 shows examples of what appears to be two different classes of $\mathrm{Ca}^{2+}$ signals generated in the trunk of embryos that could be detected and distinguished from each other, in representative aeq-mRNA injected (Fig. 6 A,B) and recombinant $f$-aequorin (Fig. $6 \mathrm{C}, \mathrm{D}$ ) injected embryos at both the 6-somite and 18-somite stages, respectively. At the 6-somite stage (Fig. $6 \mathrm{~A}, \mathrm{C}$ ), a highly localized $\mathrm{Ca}^{2+}$ transient with a duration between 60 to 120 seconds occurs in the trunk $(n=9)$, the temporal and spatial characteristics of which appear to correlate with the formation of the next somite. Similar somitogenic $\mathrm{Ca}^{2+}$ signals have been previously reported during the Segmentation Period in aequorin-injected zebrafish (Créton et al., 1998). On the other hand, at the 18-20-somite stage (Fig. 6 B,D), a more widespread, longer lasting (i.e., 10 $\mathrm{min}$ ) $\mathrm{Ca}^{2+}$ transient was observed, generated by the $\sim 12-14$ anterior-most somites alone (i.e., absent from the 6 posterior-most somites). The $\mathrm{Ca}^{2+}$ signal in the trunk of the aeq-mRNA-injected embryo at the 18-20-somite stage is shown in more detail in Fig. 7 and clearly indicates that there is sufficient aequorin reconstituted in the cells of the trunk to sustain a $\mathrm{Ca}^{2+}$ transient that lasts for at least 10 minutes. This trunk signal was seen in a consistent and reliable manner $(n=10)$ and the embryos generating them developed normally. The developmental and physiological functions of this later signal have yet to be determined. It has been reported, however, that at around this developmental stage the first muscle contractions begin in individual myotomes (Kimmel etal., 1995; Saint-Amant and Drapeau, 1998). The duration of this signal clearly indicates that it is not likely to be associated with a single muscle twitch. The onset of these contractions, however, has been reported to be correlated with axonal ingrowth into the developing muscle from the first primary motorneurons (Myers et al., 1986). Thus, this sustained trunk $\mathrm{Ca}^{2+}$ transient may play a role in some as yet poorly understood aspect of muscle development that occurs over an equivalent time frame.

Figure 8A illustrates the $\mathrm{Ca}^{2+}$ signals observed in aeq-mRNAand recombinant $f$-aequorin-injected embryos from 27-36 hpf. The level of luminescence produced by the representative $f$ aequorin-injected embryo was minimal (i.e., running at around 45 photons/sec for much of the 6-h imaging period) and failed to register any $\mathrm{Ca}^{2+}$ spiking activity. This represents $\sim 0.02 \%$ of the total amount of luminescence emitted during the entire imaging window. On the other hand, the aeq-mRNA-injected embryo exhibited both a far greater overall level of luminescence (i.e., approximately $75-100$ photons/sec) as well as a more complex pattern of $\mathrm{Ca}^{2+}$ spiking. When visualized, each individual spike represents a rapid $\mathrm{Ca}^{2+}$ transient lasting in the order of a few seconds only. We suggest that this series of $\mathrm{Ca}^{2+}$ spikes represent a third class of trunk-generated $\mathrm{Ca}^{2+}$ transients and may result from the more rapid contractions that normally result from touch-based responses during this period of zebrafish development (Saint-Amant and Drapeau, 1998). Figure 8B illustrates a representative example $(n=5)$ of another form of trunk $\mathrm{Ca}^{2+}$ transient that was generated at $\sim 47 \mathrm{hpf}$. These transients had a duration of $\sim 60 \mathrm{sec}$ and would thus appear to be of a different class again to those illustrated in Figs. 7,8A. They appear to be somewhat similar in duration to the $\sim 60$ to 120 -sec duration $\mathrm{Ca}^{2+}$ transients that have been reported to be generated in intact 
myotome isolated from stage $23 / 24$ (i.e., 25 to 26 hpf) Xenopusembryos and shown to be essential for myofibril organization and the formation of sarcomeres (Ferrari and Spitzer, 1999).

Although the precise developmental or physiological function of the trunk-generated $\mathrm{Ca}^{2+}$ transients illustrated in Figs. 6 to 8 are far from certain, they clearly indicate the potential of transiently expressing apoaequorin in zebrafish as an in situ $\mathrm{Ca}^{2+}$ imaging tool.

\section{Conclusions}

As shown by the time-course Western blot analysis (see Fig. 4), the expression of apoaequorin in the aeq-mRNA injected embryos ceased after 24 hours. This would suggest that there is a definitive limit to the amount of apoaequorin protein made in these embryos. However, experiments like the one illustrated in Fig. 8B indicate that $\mathrm{Ca}^{2+}$ signals can still be detected up to $\sim 48 \mathrm{hpf}$. This may be due to (1) a stable aequorin complex being formed, which is more resistant to the action of cellular proteases, once $f$ coelenterazine binds to apoaequorin (Shimomura, 1991) and (2) the fact that the resting level of $\mathrm{Ca}^{2+}$ within quiescent cells of a zebrafish embryo have been reported to be low, i.e., around $60 \mathrm{nM}$ (Créton et al., 1998), resulting in the finite quantities of reconstituted aequorin being used up at a very low rate (i.e., when cells are not generating $\mathrm{Ca}^{2+}$ signals).

There would appear to be two possible drawbacks to using the transient expression approach. Firstly, it cannot be used for imaging $\mathrm{Ca}^{2+}$ in embryos at stages before the apoaequorin protein is expressed in sufficient quantities to allow for detectable signal after reconstitution (i.e., before $~ 2.5 \mathrm{hpf}$, the 256cell stage). This problem can be overcome by simply injecting aequorin at the one cell stage. The second drawback results from the ubiquitous nature of mRNA-derived expression. Between 2.5 and 48 hpf (i.e., the imaging window of this technique) the three-dimensional complexity of the embryo increases dramatically, especially following gastrulation. Thus, it will become more challenging to determine precisely which group of cells, tissue or organ anlage might be responsible for generating a particular signal in later stage embryos. We plan to resolve this shortcoming in the future by developing stable lines of transgenic zebrafish that both express apoaequorin in a tissue-specific manner and further extend the $\mathrm{Ca}^{2+}$-imaging window.

Nevertheless, we believe that the transient expression approach is a valuable interim tool (that can be used while the transgenic lines are being generated), for monitoring $\mathrm{Ca}^{2+}$ signaling events that occur during the relative later stages of zebrafish development (i.e., 24 to $48 \mathrm{hpf}$ ), when injected aequorin has been used up. This effectively extends the aequorin-based $\mathrm{Ca}^{2+}$ imaging period by an additional 24 hours. Thus, in combination with injecting aequorin at the single-cell stage, $\mathrm{Ca}^{2+}$ signaling in zebrafish can be now continuously monitored during development from fertilization up to the long pec stage, at $48 \mathrm{hpf}$. We suggest, therefore, that this method represents a significant step forward in the aequorin-based imaging technique by providing a straightforward and rapid first step for the study of developmental $\mathrm{Ca}^{2+}$ signals in later-stage zebrafish embryos. It also provides an alternative method to generating transgenic zebrafish lines that express $\mathrm{Ca}^{2+}$ probes based on fluorescent proteins such as cameleons (Higashima et al., 2003).

\section{Experimental Procedures}

\section{Embryo collection}

Zebrafish (Danio rerio), AB strain, were maintained on a $14 \mathrm{hr}$ light/10 hr dark cycle to stimulate spawning (Westerfield, 1994) and their fertilized eggs were collected as described elsewhere (Webb et al., 1997). Embryos were placed in a custom-designed holding/imaging chamber, 
described in detail in Webb et al. (1997), in 30\% Danieau's solution (17.4 $\mathrm{mM} \mathrm{NaCl}, 0.21 \mathrm{mM} \mathrm{KCl}, 0.18 \mathrm{mM} \mathrm{Ca}\left(\mathrm{NO}_{3}\right)_{2}, 0.12 \mathrm{mM} \mathrm{MgSO}_{4} .7 \mathrm{H}_{2} \mathrm{O}, 1.5$ $\mathrm{mM}$ Hepes, $\mathrm{pH} 7.2$ ) and maintained at $\sim 28.5^{\circ} \mathrm{C}$ throughout development.

\section{Preparation of the pSP64TNE-aeq and pSP64TNE-aeq::EGFP plas- mids}

The apoaequorin plasmid (piP-HE; Inouye et al., 1989) was obtained from Prof. Frederick I. Tsuji (Scripps Institution of Oceanography, University of California San Diego) and the pSP64T-derived plasmid (Kreig and Melton, 1984), pSP64TNE, was obtained from Prof. K.L. Chow (The Hong Kong University of Science and Technology, Hong Kong). All the restriction enzymes were from New England Biolabs Inc. Two plasmids were made; (1) pSP64TNE-aeq, containing the apoaequorin sequence and (2) pSP64TNE-aeq::EGFP, containing a fusion protein of apoaequorin and EGFP (Fig. 1).

For the pSP64TNE-aeq plasmid, PCR was carried out to amplify the apoaequorin sequence (591 bp) from the piP-HE plasmid, using the following oligonucleotide primers:

5'-accagcggccgcatgacaagcaaacaatactcagtcaagcttacatcagac-3' and

5'-accagaattcttaggggacagctccaccgtagag-3', in which two restriction sites, Not and ECOR1, were added to the 5' and 3' ends of the apoaequorin sequence, respectively. The apoaequorin sequence was then cloned into the pSP64TNE plasmid using these restriction sites.

For the pSP64TNE-aeq::EGFP plasmid, the apoaequorin sequence was amplified from piP-HE using the

5'-accaggtaccatgacaagcaaacaatactcagtcaagcttacatcagac-3' and

5'-accaaccggtatggggacagctccaccgtagag-3' primers, in which the stop codon was deleted and two restriction sites, Kpn1 and Age1, were added to the 5' and 3' ends, respectively. The apoaequorin sequence was then cloned into the pSP64TNE-EGFP plasmid (the EGFP sequence was subcloned from pEGFP-N1; Clontech) using the Kpn1 and Age1 restriction sites and fused in-frame at the 5' end of EGFP. Further details of the constructs used are available upon request.

\section{In vitro synthesis of aeq-mRNA and aeq::EGFP-mRNA and microin- jection}

The pSP64TNE-aeq and pSP64TNE-aeq::EGFP plasmids were linearized with Xba1. Apoaequorin (aeq-mRNA) and apoaequorin-EGFP (aeq.:EGFP-mRNA) transcripts were made and precipitated using the mMESSAGE mMACHINE ${ }^{\circledR}$ sp6 Kit (Ambion) following the protocol recommended by the manufacturer.

Embryos were injected with $400 \mathrm{pg}$ aeq::EGFP-mRNA into the blastodisc at the 1-cell stage. The microinjection pipettes and pressure injection system used are described in detail elsewhere (Webb et al., 1997). Brightfield and fluorescence images of embryos at the 256-cell, 12-somite and 20-somite stages, were captured with a Nikon DXM 1200F camera mounted on a Zeiss Axioskop using a Zeiss Plan Neofluar 10x/0.3NA objective. GFP fluorescence was captured using $488 \mathrm{~nm}$ excitation/ $507 \mathrm{~nm}$ emission.

\section{Testing mRNA toxicity}

Different amounts of aeq-mRNA (i.e., $500 \mathrm{pg}, 700 \mathrm{pg}$ and $1500 \mathrm{pg}$ per embryo) and aeq.:EGFP-mRNA (i.e., 400 pg, 500 pg, $700 \mathrm{pg}$ and $1500 \mathrm{pg}$ per embryo), were microinjected into the blastodisc of embryos at the 1-cell stage. The mRNA injected embryos were observed at $24 \mathrm{hpf}$ and brightfield images were captured using the Nikon DXM 1200F camera and Zeiss 10x objective described earlier.

\section{Western blot analysis}

Embryos that had been injected with 700 pg aeq-mRNA or $400 \mathrm{pg}$ aeq.:EGFP-mRNA at the 1-cell stage were used for Western blot analysis at 2, 4, 8, 12, 18, 24 and $30 \mathrm{hpf}$. Embryos without mRNA injection were also prepared as a negative control. The chorions and yolks of two embryos per time-point were manually removed using watchmaker's forceps in cold Ringer's buffer (Westerfield, 1994) containing protease inhibitor (1 tablet/ $50 \mathrm{ml}$; Roche) and then discarded. The remaining embryonic tissues were then transferred together with cold Ringer's buffer to an equivalent volume of $2 \mathrm{X}$ sample buffer $(300 \mathrm{mM} \mathrm{NaCl}, 2 \% \mathrm{NP}-40,1 \%$ Triton $\mathrm{X}-100,1 \%$ deoxycholic acid, $0.2 \%$ SDS, $100 \mathrm{mM}$ Tris- $\mathrm{HCl}, 2 \mathrm{mM}$ EDTA and $2 \mathrm{mM}$ EGTA). The protein in the samples was denatured at $75-80^{\circ} \mathrm{C}$ with $5 \mathrm{X}$ loading buffer $(0.313 \mathrm{M} \mathrm{Tris} \mathrm{HCl}, 50 \%$ glycine, $10 \%$ SDS, $0.05 \%$ bromophenol blue and 10\% 2-mercaptoethanol) and then resolved with a $10 \%$ SDSpolyacrylamide gel (consisting of a Separating gel: $375 \mathrm{mM}$ Tris base, $0.1 \%$ SDS (pH 8.8), $10 \%$ acrylamide, $0.15 \%$ APS and $0.001 \%$ TEMED; and Stacking gel: $125 \mathrm{mM}$ Tris base, $0.1 \%$ SDS (pH 6.8), $4 \%$ acrylamide, $0.1 \%$ APS and $0.002 \%$ TEMED). The SDS-PAGE was carried out in running buffer (25 mM Tris-base, $190 \mathrm{mM}$ glycine and $0.1 \%$ SDS) at $120 \mathrm{~V}$ using a Mini-Protean ${ }^{\circledR} 3$ Cell (Bio-rad). Recombinant $f$-aequorin (20 pg; Shimomura etal., 1989; The Photoprotein Laboratory, Falmouth, MA, USA) was loaded into one lane of the gel as an internal positive control.

The separated proteins were then transferred to a nitrocellulose membrane (Bio-rad) overnight at $4^{\circ} \mathrm{C}$ in cold transfer buffer $(2.4 \mathrm{mM} \mathrm{NaOAc}, 10$ $\mathrm{mM}$ MOPS, $20 \%$ ethanol, $0.1 \%$ SDS) using a Mini Trans-Blot ${ }^{\circledR}$ Electrophoretic Transfer Cell (Bio-rad) set at 20 V. For detection of apoaequorin, the nitrocellulose membrane was incubated with blocking buffer (PBS; $136.9 \mathrm{mM} \mathrm{NaCl}, 2.7 \mathrm{mM} \mathrm{KCl}, 32 \mathrm{mM} \mathrm{Na}_{2} \mathrm{HPO}_{4}$ and $8 \mathrm{mM} \mathrm{NaH}_{2} \mathrm{PO}_{4}, \mathrm{pH} 7.3$; containing $0.1 \%$ Triton $\mathrm{X}-100$ and $10 \%$ goat serum) for $1 \mathrm{~h}$ and then probed with a rabbit polyclonal antibody against aequorin (Abcam; at a dilution of 1:2,000 in blocking buffer) for $1 \mathrm{~h}$. Following $4 \times 10$-min washes in PBS containing $0.1 \%$ Triton $X-100$, the membrane was blocked again, this time for 20 min with blocking buffer, after which it was incubated with a horseradish peroxidase conjugated goat anti-rabbit IgG secondary antibody (Santa Cruz; at a dilution of 1:20,000 in blocking buffer) for $1 \mathrm{~h}$. The membrane was washed for $6 \times 10$ min with PBS containing $0.1 \%$ Triton X100 and then the presence of apoaequorin protein was visualized with the SuperSignal ${ }^{\circledR}$ West Pico Chemiluminescent Substrate (Pierce) and exposed on the Kodak BioMax Light-1 film (Amersham) for 1 min. Signals on the film were quantified using NIH Image $\mathrm{J}$ and normalized using the recombinant aequorin (internal positive control) band, prior to graph plotting.

\section{Preparation of $\mathrm{f}$-coelenterazine}

A stock solution of $2 \mathrm{mg} / \mathrm{ml} f$ coelenterazine (Molecular Probes) was prepared in methanol. Just prior to use, this stock solution was diluted to either $2 \mu \mathrm{M}$ in homogenization buffer $(20 \mathrm{mM}$ Tris $\mathrm{HCl}, 10 \mathrm{mM}$ EDTA, $10 \mathrm{mM}$ EGTA, pH 7.5) for the in vitroaequorin reconstitution assay, or to $50 \mu \mathrm{M}$ in $30 \%$ Danieau's solution for in vivo aequorin reconstitution.

\section{In vitro aequorin reconstitution assay}

Embryos $(n=10)$ that had been injected with $700 \mathrm{pg}$ aeq-mRNA or 400 pg aeq.:EGFP-mRNA at the 1-cell stage were manually dechorionated at either $4 \mathrm{hpf}$ or $24 \mathrm{hpf}$ using watchmaker's forceps. The dechorionated embryos were then homogenized for $10-20 \mathrm{sec}$ in $0.5 \mathrm{ml}$ homogenization buffer containing protease inhibitor ( 1 tablet $/ 50 \mathrm{ml}$ of buffer; Roche), using a sonicator. Extract prepared from uninjected embryos was used as a negative control.

Aequorin luminescence was acquired using an FB15 luminometer (Zylux Corp.). The embryo extract was transferred to a $12 \times 75 \mathrm{~mm}$ disposable borosilicate glass culture tube and $0.5 \mathrm{ml}$ of $2 \mu \mathrm{M} f$ coelenterazine was added for the reconstitution of apoaequorin to aequorin. After reconstitution for $1 \mathrm{~h}, 200 \mu \mathrm{l}$ of $150 \mathrm{mM} \mathrm{CaCl}$ was added to the tube via the automatic injector of the luminometer. The level of luminescence (measured in relative light units, RLU) was then accumulated for 1 min after adding the $\mathrm{CaCl}_{2}$. The values of total luminescence output were then transferred to Excel 2000 (Microsoft) for data processing and graph plotting.

\section{In vivo reconstitution of aequorin}

Embryos were microinjected with $700 \mathrm{pg}$ aeqmRNA into the center of the blastodisc at the 1 -cell stage and were then maintained at $\sim 28.5^{\circ} \mathrm{C}$ until the 64-cell stage. The embryos were then dechorionated manually with 
watchmaker's forceps, after which they were transferred to a holding/ imaging chamber and then incubated with $50 \mu \mathrm{M} f$ coelenterazine in $30 \%$ Danieau's solution. Embryos bathed in this $f$-coelenterazine solution were then transferred to a custom-built Photon Imaging Microscope System (PIMS; Science Wares, East Falmouth, MA, USA) for data acquisition. An outline of our PIMS and the photon acquisition procedure is described in detail elsewhere (Webb et al., 1997).

\section{Injection of $\mathrm{f}$-aequorin}

The protocols used for injecting embryos with aequorin are described in detail elsewhere (Webb et al., 1997). Approximately $4 \mathrm{nl}$ of recombinant $f$ aequorin (at $\sim 1 \%$ in $100 \mathrm{mM} \mathrm{KCl}, 5 \mathrm{mM}$ MOPS and $50 \mu \mathrm{M}$ EDTA) was injected into the top of the embryo's yolk cell at the 128-cell stage. Following microinjection, embryos were transferred to our PIMS for data acquisition.

\section{Acknowledgements}

We thank Frederick I. Tsuji for the piP-HE apoaequorin plasmid and King Lau Chow for the pSP64TNE plasmid. Special thanks to Drs. O. Shimomura, Y. KishiandS. Inouye for supplying us with thef-aequorin. The work was supported by a joint National Natural Science Foundation of Chinal Hong Kong Research Grants Council grant, with grant numbers N HKUST607/01 and 30131160732, awarded to A.L.M. and A.M., respectively and by RGC grants HKUST6214/02M, HKUST6279/03M and HKUST6241/04M awarded to A.L.M and S.E.W. and was carried out while A.L.M. was the recipient of a Croucher Senior Research Fellowship. The zebrafish were supplied by the Zebrafish International Resource Center, supported by grant \#RR12546 from the NIH-NCRR.

\section{References}

ASHWORTH, R. and BRENNAN, C. (2005). Use of transgenic zebrafish reporter lines to study calcium signaling in development. Brief. Func. Gen. Proteom. 4: 186-193.

BAUBET, V., LE MOUELLIC, H., CAMPBELL, A.K., LUCAS-MEUNIER, E., FOSSIER, P. and BRULET, P. (2000). Chimeric green fluorescent protein-aequorin as bioluminescent $\mathrm{Ca}^{2+}$ reporters at the single-cell level. Proc. Natl. Acad. Sci. USA 97: 7260-7265.

BERRIDGE, M.J., BOOTMAN, M.D. and RODERICK, H.L. (2003). Calcium signalling: dynamics, homeostasis and remodeling. Nat. Rev. Mol. Cell. Biol. 4: 517-529.

BOOTMAN, M.D., COLLINS, T.J., PEPPIATT, C.M., PROTHERO, L.S., MACKENZIE, L., DE SMET, P., TRAVERS, M., TOVEY, S.C., SEO, J.T., BERRIDGE, M.J., CICCOLINI, F. and LIPP, P. (2001). Calcium signalling-an overview. Semin. Cell. Dev. Biol. 12: 3-10.

BUTTON, D. and BROWNSTEIN, M. (1993). Aequorin-expressing mammalian cell lines used to report $\mathrm{Ca}^{2+}$ mobilization. Cel/ Calcium 14: 663-671.

CRÉTON, R., STEELE, M.E. and JAFFE, L.F. (1997). Expression of apo-aequorin during embryonic development; how much is needed for calcium imaging? Cell Calcium 22: 439-446.

CRÉTON, R., SPEKSNIJDER, J.E. and JAFFE, L.F. (1998). Patterns of free calcium in zebrafish embryos. J. Cell. Sci. 111: 1613-1622.

FERRARI, M.B. and SPITZER, N.C. (1999). Calcium signaling in the developing Xenopus myotome. Dev. Biol. 213: 269-282.

GILLAND, E., MILLER, A.L., KARPLUS, E., BAKER, R. and WEBB, S.E. (1999). Imaging of multicellular large-scale rhythmic calcium waves during zebrafish gastrulation. Proc. Natl. Acad. Sci. USA 96: 157-161.

GILMOUR, D.T., JESSEN, J.R. and LIN, S. (2002). Manipulating gene expression in the Zebrafish. In Zebrafish: A practical approach (C. Nüsslein-Volhard and R. Dahm, Eds.) pp.121-143. Oxford University Press, Oxford.

HEAD, J.F., INOUYE, S., TERANISHI, K. and SHIMOMURA, O. (2000). The crystal structure of the photoprotein aequorin at $2.3 \AA$ resolution. Nature 405: 372-376.

HIGASHIJIMA, S., MASINO, M.A., MANDEL, G. and FETCHO, J.R. (2003). Imaging neuronal activity during zebrafish behavior with a genetically encoded calcium indicator. J. NeuroPhysiol. 90: 3986-3997.
INOUYE, S., AOYAMA, S., MIYATA, T., TSUJI, F.I. and SAKAKI, Y. (1989). Overexpression and purification of the recombinant $\mathrm{Ca}^{2+}$ binding protein, apoaequorin. $\mathrm{J}$. Biochem. 105: 473-477.

KIMMEL, C.B., BALLARD, W.W., KIMMEL, S.R., ULLMANN, B. and SCHILLING, T.F. (1995). Stages of Embryonic Development of the Zebrafish. Dev. Dyn. 203: 253310.

KRIEG, P.A. and MELTON, D.A. (1984). Functional messenger RNAs are produced by SP6 in vitro transcription of cloned cDNAs. Nuc. Acid Res. 12: 7057-7070.

LEE, K.W., WEBB, S.E. and MILLER, A.L. (1999). A wave of free cytosolic calcium traverses zebrafish eggs on activation. Dev. Biol. 214: 168-180.

LEUNG, C.F., WEBB, S.E. and MILLER, A.L. (1998). Calcium transients accompany ooplasmic segregation in zebrafish embryos. Dev. Growth Differ. 40: 313-326.

MARCH, J.C., RAO, G. and BENTLEY, W.E. (2003). Biotechnological applications of green fluorescent protein. Appl. Microbiol. Biotechnol. 62: 303-315.

MAZIA, D. (1937). The release of calcium in Arbaciaeggs on fertilization. J. Cell Comp. Physiol. 10: 291-304.

MILLER, A.L., KARPLUS, E. and JAFFE, L.F. (1994). Imaging $\left[\mathrm{Ca}^{2+}\right]$ i with aequorin using a photon imaging detector, In Methods in Cell Biology (R. Nuccitelli, Ed.) Vol. 40, pp. 305-338. Academic Press, San Diego, CA.

MYERS, P.Z., EISEN, J.S. and WESTERFIELD, M. (1986). Development and axonal outgrowth of identified motoneurons in the zebrafish. J. Neurosci. 6: 2278-2289.

RIDGWAY, E.B. and ASHLEY, C.C. (1967). Calcium transients in single muscle fibers. Biochem. Biophys. Res. Commun. 29: 229-234.

SAINT-AMANT, L. AND DRAPEAU, P. (1998). Time course of the development of motor behaviors in the zebrafish embryo. J. Neurobiol. 37: 622-632.

SARAN, S., NAKAO, H., TASAKA, M., IIDA, H., TSUJI, F.I., NANJUNDIAH, V. and TAKEUCHI, I. (1994). Intracellular free calcium level and its response to cAMP stimulation in developing Dictyosteliumcells transformed with jellyfish apoaequorin cDNA. FEBS Lett. 337: 43-47.

SHIMOMURA, O. (1991). Preparation and handling of aequorin solution for the measurement of cellular $\mathrm{Ca}^{2+}$. Cell Calcium 12: 635-643.

SHIMOMURA, O. (1995). Cause of spectral variation in the luminescence of semisynthetic aequorins. Biochem. J. 306: 537-543.

SHIMOMURA, O. (1997). Membrane permeability of coelenterazine analogues measured with fish eggs. Biochem. J. 326: 297-298.

SHIMOMURA, O., MUSICKI, B. and KISHI, Y. (1989). Semi-synthetic aequorins with improved sensitivity to $\mathrm{Ca}^{2+}$ ions. Biochem. J. 261: 913-920.

SHIMOMURA, O. and INOUYE, S. (1996). Titration of recombinant aequorin with calcium chloride. Biochem. Biophys. Res. Communs. 221: 77-81.

SHIMOMURA, O., JOHNSON, F.H. and SAIGA, Y. (1962). Extraction, purification and properties of aequorin, a bioluminescent protein from the luminous hydromedusan, Aequorea. J. Cell. Comp. Physiol. 59: 223-239.

SHIMOMURA, O. and JOHNSON, F.H. (1978). Peroxidized coelenterazine, the active group in the photoprotein aequorin. Proc. Natl. Acad. Sci. USA 75: 26112615.

SHIMOMURA, O. and JOHNSON, F.H. (1973). Chemical nature of light emitter in bioluminescence of aequorin. Tetra. Lett. 31: 2963-2966.

SHIMOMURA, O. and JOHNSON, F.H. (1975). Regeneration of the photoprotein aequorin. Nature 256: 236-238.

WEBB, S.E., LEE, K.W., KARPLUS, E. and MILLER, A.L. (1997). Localized calcium transients accompany furrow positioning, propagation and deepening during the early cleavage period of zebrafish embryos. Dev. Biol. 192: 78-92.

WEBB, S.E. and MILLER, A.L. (2003). Calcium signalling during embryonic development. Nat. Rev. Mol. Cell Biol. 4: 539-551.

WESTERFIELD, M. (1994). "The Zebrafish Book: A Guide for the Laboratory Use of Zebrafish (Brachydanio rerio)". Univ. of Oregon Press, Eugene, OR.

Received: January 2006 Reviewed by Referees: April 2006 Modified by Authors and Accepted for Publication: May 2006 Published Online: May 2006 